\title{
Radon Exhalation Patterns in A Dead-end Tunnel
}

\author{
Shu Guang WANG ${ }^{a}$, Xiao Bing NIU, Qing CHEN, Wei YANG \\ Northwest Institute of Nuclear Technology, Shaanxi, Xi'an, 710024, China
}

\begin{abstract}
The regularity of radon exhalation rate in the over-broken granite tunnel is susceptible to weather conditions and ventilation styles. Based on the calculation model of radon exhalation in tunnel, some experiments have been carried out to analyze the variations of radon exhalation in cases of natural ventilation, blowing ventilation and exhaust ventilation separately. The results show that there is a linear relation between the radon exhalation and the natural ventilation quantity, and also between the radon exhalation and the ambient temperature; the radon exhalation in the case of exhaust ventilation is $63 \%$ higher than that in the blowing case under the condition of the same ventilation quantity and ambient temperature. Therefore, it is suggested that operation in the tunnel in high temperature be avoided in summer, and the blowing ventilation be adopted as an effective way for ventilation.
\end{abstract}

\section{Introduction}

In addition to the physical properties of rock and the exposed area of surrounding rock, the main factors affecting the radon exhalation rate in tunnel include the weather conditions and ventilation styles. As early as 1951, Smith, O. F. discovered the effects of weather conditions on the soil radon exhalation; in 1963, Schroeder, G. L. began to test the control of radon exhalation during the tunnel construction in pressurized ventilation $^{[1]}$. F. Perrier ${ }^{[2]}$ et al, through on-site monitoring of the tunnel with the length of $38 \mathrm{~m}$ and slope of $8 \%$ in Pulchoki Mount of Nepal, achieved the following conclusions: obvious natural wind only existed in this tunnel in winter (from October to next March, the temperature outside the tunnel is lower than that inside) and the radon concentration in the tunnel changed with the ventilation quantity. Perrier ${ }^{[2]}$ pointed out that the change in radon concentration was very sensitive to airflow exchange, but he did not make a quantitative description of the changing relationship between them. Mechanical ventilation had been widely used as a common way of radon reduction in underground construction $^{[1]}$, but the relationship between mechanical ventilation and radon exhalation rate was still unclear.

Through experiments, this paper studies the regularity of radon exhalation respectively in natural ventilation and mechanical ventilation in an over-broken granite dead-end tunnel in Northwest China, and analyzes the effects of outside temperature on the radon exhalation in tunnel in different ventilation cases. The pollution of radon in tunnel in summer is the most serious, so this paper just focuses on the situation in summer.

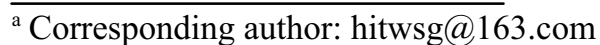

\section{Formula of Radon Exhalation Rate in Ventilated Tunnel}

The calculation model of change of concentration of radon in the ventilated tunnel is shown in Fig.1. Take the micro unit of $d x$ wide (right in Fig.1), then at a certain time, the equilibrium equation of radon in the micro unit radon is:

$$
C_{1} Q_{1}+\delta s d x=C_{2} Q_{2}+\lambda A d x C_{1}
$$
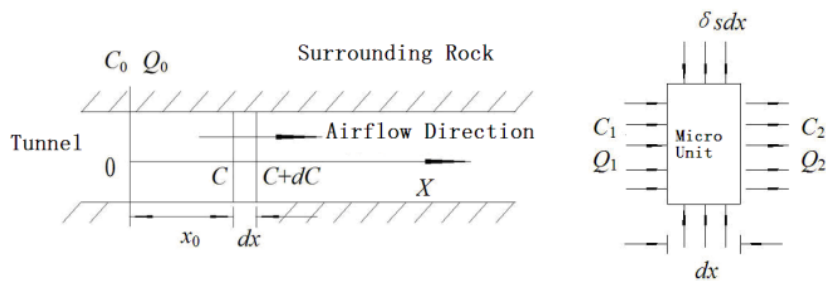

a. Ventilated Tunnel

b. Micro Unit

Fig.1 Calculation model of radon concentration in the ventilated tunnel

Where: $C_{1}$ is the concentration of radon flowing into the micro-unit airflow, $\mathrm{Bq} / \mathrm{m}^{3} ; Q_{1}$ is the volume of air flowing into the micro unit, $\mathrm{m}^{3} / \mathrm{s} ; \delta$ is the radon exhalation rate on the surrounding rock surface, $\mathrm{Bq} /\left(\mathrm{s} \cdot \mathrm{m}^{2}\right) ; s$ is the sectional perimeter of tunnel, $\mathrm{m} ; A$ is the cross-sectional area of tunnel, $\mathrm{m}^{2} ; d x$ is the width of micro unit, $\mathrm{m} ; C_{2}$ is the concentration of radon flowing out of micro unit, $\mathrm{Bq} / \mathrm{m}^{3} ; Q_{2}$ is the volume of air flowing out of micro unit, $\mathrm{m}^{3} / \mathrm{s} ; \lambda$ is the radon decay constant, $\mathrm{s}^{-1}$.

The physical meaning of Eq.1: at a certain time, the sum of the amount of radon $C_{1} Q_{1}$ flowing into the micro 
unit by the tunnel airflow and the amount of radon $\delta s d x$ exhaled from the surrounding rock surface to the micro unit is equal to the sum of the amount of radon $C_{2} Q_{2}$ flowing out of the micro unit and the decay amount of radon $\lambda A d x C_{l}$ in the micro unit.

Also, $C_{1}$ is equal to $C$ shown at the left of Fig., $Q_{1}$ is equal to $Q_{0}+q x_{0}, C_{2}$ is equal to $C+d C, Q_{2}$ is equal to $Q_{0}+q$ $\left(x_{0}+d x\right)$. Therefore, the Eq. 1 can be rewritten as

$$
\delta s d x+C\left(Q_{0}+q x_{0}\right)=\left[Q_{0}+q\left(x_{0}+d x\right)\right](C+d C)+\lambda A C d x
$$

Where, $C$ is the radon concentration at the coordinates $x_{0}, \mathrm{~Bq} / \mathrm{m} 3 ; q$ is the natural ventilation quantity of tunnel with per unit length, $\mathrm{m}^{3} /(\mathrm{m} \cdot \mathrm{s}) ; Q_{0}$ is the ventilation quantity of tunnel at the origin of coordinates, $\left(\mathrm{m}^{3} / \mathrm{s}\right) ; \delta$ is radon exhalation rate of surrounding rock, $\mathrm{Bq} /\left(\mathrm{s} \cdot \mathrm{m}^{2}\right) ; s$ is the sectional perimeter of tunnel, taking the average of $12 \mathrm{~m} ; A$ is cross-sectional area of tunnel, taking the design value of $9 \mathrm{~m}^{2}$.

Omit the second-order infinitesimal and take the radon exhalation rate $\delta$ as a constant, then Eq. 2 is changed as

$$
\frac{d C}{\delta s-\lambda A C-q C}=\frac{d x}{Q_{0}+q x_{0}}
$$

Substitute the boundary condition $\left.C\right|_{X=0}=C_{0}$, then the solution of the equation is:

$$
C=\frac{\delta s}{\lambda A+q}+\left(C_{0}-\frac{\delta s}{\lambda A+q}\right)\left(\frac{Q_{0}}{Q_{0}+q x_{0}}\right)^{\frac{\lambda A+q}{q}}
$$

After deformation, the above equation is:

$$
\delta=\frac{(\lambda A+q)\left[C-C_{0}\left(\frac{Q_{0}}{Q_{0}+q x_{0}}\right)^{\frac{\lambda A+q}{q}}\right]}{s\left[1-\left(\frac{Q_{0}}{Q_{0}+q x_{0}}\right)^{\frac{\lambda A+q}{q}}\right]}
$$

The actual measured $q$ of the tunnel is approximately $10^{-4} \mathrm{~m}^{3} /(\mathrm{s} \cdot \mathrm{m})$, and $\lambda A$ is about $10^{-5} \mathrm{~m}^{2} / \mathrm{s}$. Therefore it can be approximately considered that $\lambda A+q \approx q$, then the Eq.3 can be simplified as:

$$
\delta=\frac{C\left(q x_{0}+Q_{0}\right)-C_{0} Q_{0}}{s x_{0}}
$$

Where, $\left(q x_{0}+Q_{0}\right)$ is the sectional ventilation quantity at the coordinate $x_{0}$.

The Eq. 4 is the formula of radon exhalation rate. As $s$ and $x_{0}$ are dimension parameters of tunnel and are the known quantities in the formula, the radon exhalation rate can be calculated by just measuring the radon concentrations $\left(C\right.$ and $\left.C_{0}\right)$ and ventilation quantities $(q$ and $Q_{0}$ ) of two sections.

\section{Impact of Natural Ventilation on Radon Exhalation Rate}

During the measurement of radon exhalation rate, according to the air state of the measured environment, the measurement methods can be divided into two kinds, namely dynamic and static methods ${ }^{[3,4]}$. The so-called dynamic method is to measure the radon exhalation rate in the case of air flow, while the static method is to do so in the still air. There is always natural ventilation in the experimented tunnel. Therefore, only the dynamic method is used for the measurement of radon exhalation rate.

\subsection{Experimental methods and measures}

The quantity of natural ventilation in tunnel is usually small and the wind speed is small, so it is not easy to conduct the all-section measurement. Meanwhile, the distribution of natural ventilation flow field is and the wind directions above and below the section are opposite (Fig. 2). In order to avoid the effects of external weather conditions on the measurement results and improve the reliability of the measurement data, an air door is set up $100 \mathrm{~m}$ away from the entry of the tunnel (Fig. 3) as the natural ventilation's section of wind speed measurement. The size of inside section is adjusted by adjusting the number of inserted door planks. The natural ventilation quantity can be calculated according to sectional wind speed and vent area. During measurement, adjust the width of air door to $13 \mathrm{~cm}$, set up 3 measurement points on the measurement section (Fig. 3 (b)) and average the sectional wind speeds. Calculate the ventilation quantity according to the sectional wind speed and ventilation $\operatorname{area}^{[5,6]}$.

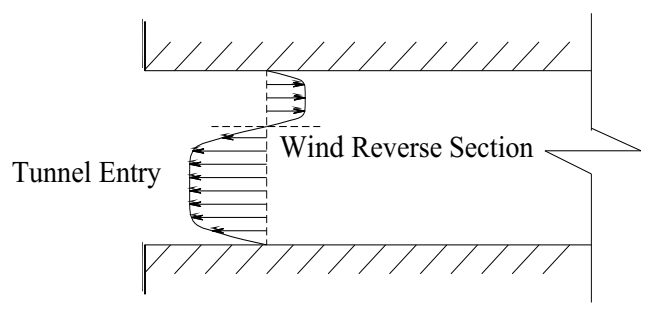

(a) Summer Conditions

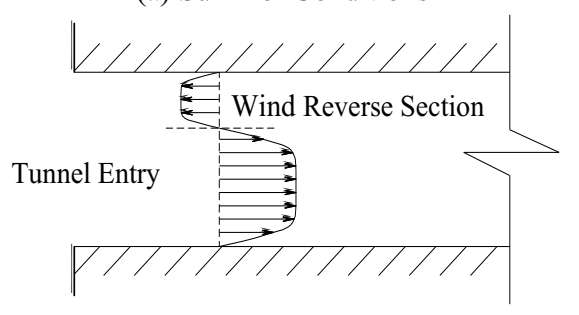

(b) Winter Conditions

Fig. 2 Seasonal change law of natural ventilation in tunnel

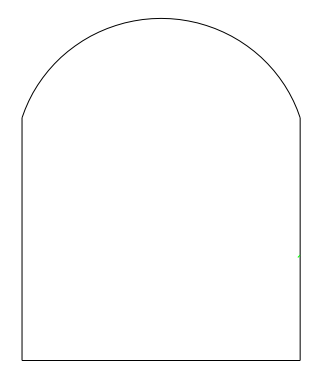

(a) Section of Original Tunnel

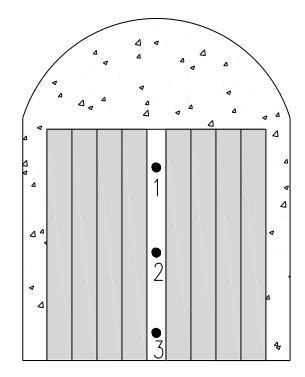

(b) Rectification Section and Measurement Points
Fig. 3 Layout of recitation section and measurement point of tunnel ventilation 


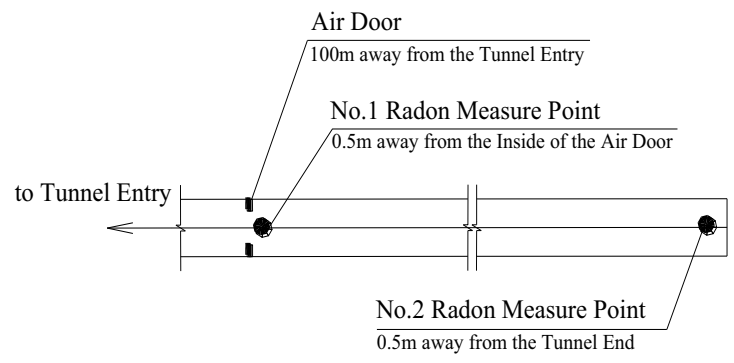

Fig. 4 Overall layout of radon exhalation ratemeasurement in natural ventilation case

The overall layout of the experiment is shown in Fig. 4. The distance from the measured tunnel section to the end of tunnel is about $600 \mathrm{~m}$. Study the regularity of radon exhalation according to the measurement principle with the dynamic method in the natural ventilation as well as Eq.4. Not only the natural ventilation quantity, surface area of tunnel and its length shall be measured, but it is necessary to monitor the concentration of radon in tunnel as well as the outside temperature.

(1) Radon concentration measurement Under the normal conditions in summer, there is a large amount of radon accumulated in the tunnel. The transfer of the radon is mainly controlled by the convection and diffusion, so that the radon concentration reaches relative balanced state in a certain time. Experimental results show that, in the absence of mechanical ventilation, the radon concentrations in the tunnel are basically the same everywhere, but the radon concentration in the rock wall is slightly higher with the presence of cracks (or rock bolt orifice). Therefore, if the effect of surrounding rock nonuniformity is ignored, and the radon concentration is measured in natural state theoretically, the radon detector can be placed in any position. The measuring method is as follows: set up two radon concentration monitoring points (Fig. 4) in the tunnel, both located in the center of the cross section of the tunnel, and average the radon concentrations at two measurement points. The main instrument used is RAD7 continuous electronic radon detector, with the accuracy of $\pm 5 \%$ (measuring range is from $3.7 \mathrm{~Bq} / \mathrm{m}^{3}$ to $7.4 \times 10^{4} \mathrm{~Bq} / \mathrm{m}^{3}$ ).

(2) Monitor temperature Set up a thermometer screen outside the tunnel and place the metrological sensor in it to continuously monitor the outside temperature. The main component used is HCD1019-5P multi- parameter metrological sensor, with the temperature measurement accuracy of $< \pm 0.3^{\circ} \mathrm{C}$ (measurement range is from -40 to $+123.8^{\circ} \mathrm{C}$ ).

\subsection{Data analysis}

\subsubsection{Natural ventilation quantity and radon exhalation rate}

Radon exhalation rate is calculated according to Eq.4. The relationship between the natural ventilation quantity and radon exhalation rate is shown in Fig.5.

After Linear regression analysis, the regression equation of radon exhalation rate and natural ventilation quantity in the natural state is:

$$
\delta=9.0765 Q-0.4854
$$

It can be seen that the radon exhalation rate is positively correlated with the natural ventilation quantity.

In theory, in the case of natural ventilation: $Q_{0}=0, q \neq$ 0 . Substitute this condition into Eq. 3 and it is obtained as follows after transformation:

$$
\delta=\frac{C}{S} \cdot Q+\frac{\lambda V C}{S}
$$

Where, $Q$ is the natural ventilation quantity, $\mathrm{m}^{3} / \mathrm{s} ; V$ is the tunnel volume, $\mathrm{m}^{3} ; S$ is the surface area of the tunnel, $S=s x, \mathrm{~m}^{2}$.

It can be seen, among the physical quantities in the Eq.6, the rest are the constants except for the natural ventilation quantity $Q$ as a variable. Namely, this equation is like the style of a unitary linear function: $y=a x+b$, indicating linear relationship between radon exhalation rate $\delta$ and the natural ventilation quantity $Q$. This conclusion is consistent with the analysis results of the above measured data.

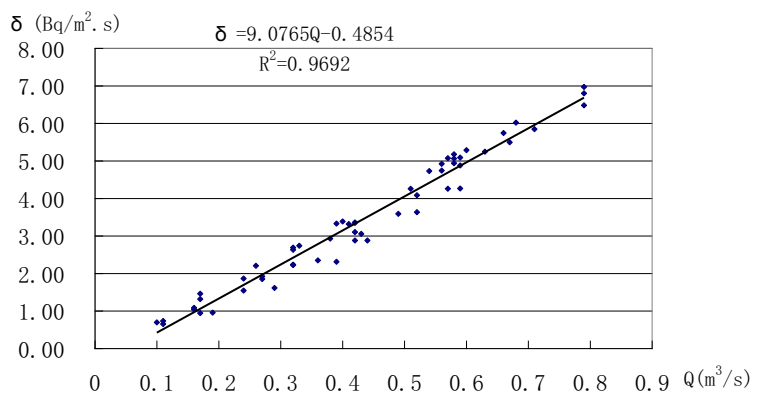

Fig. 5 Relationship between natural ventilation quantity and radon exhalation rate

\subsubsection{Correlation analysis of radon exhalation rate and temperature}

(1) Regression equation Based on the mechanism of radon exhalation, the key factor affecting the radon exhalation rate is the pressure difference between the inside and outside of the tunnel and the pressure difference is due to the temperature change. Therefore, the study of relationship between the environmental temperature and radon exhalation rate shall be emphasized. Effective data of a total of 56 groups is obtained in the experiment. The scatter diagram of temperature and radon exhalation rate is shown in Fig. 6.

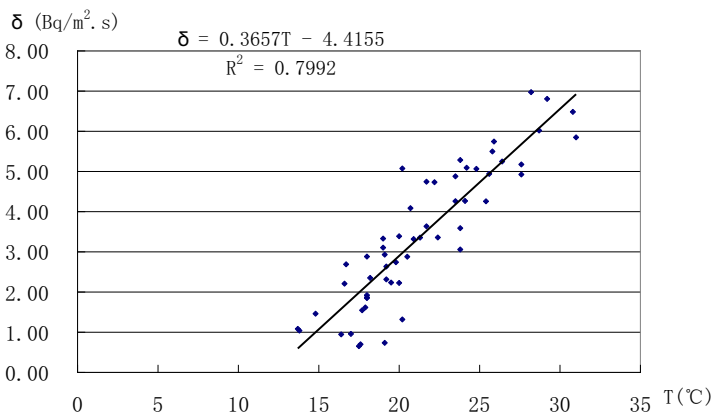

Fig. 6 The outside temperature VS radon exhalation rate in natural ventilation case 
Fig.6 shows that there is an obvious linear relationship between temperature and radon exhalation rate. After the linear regression analysis, the linear regression equation of radon exhalation rate and temperature is:

$$
\delta=0.3657 T-4.4155
$$

Where, $T$ is the temperature outside the tunnel, ${ }^{\circ} \mathrm{C}$.

(2) Test of linear regression significance Test the significance of linear regression with the $F$ testing method:

$$
F=(n-2) \frac{R^{2}}{1-R^{2}}=214.92
$$

Look up the table of $F$ distribution critical values and obtain: when $\alpha=0.05$, the degree of freedom is ( 1 , $n-2)$, namely the critical value $\lambda$ of $(1,54)$ is $4.00<\lambda_{\alpha=0.05}<4.08$, then $F>\lambda_{\alpha=0.05}$, namely the linear regression is significant. Look up the table of $F$ distribution critical values and obtain: when $\alpha=0.01$, the degree of freedom is $(1, n-2)$, namely the critical value $\lambda$ of $(1,54)$ is $7.08<\lambda_{\alpha=0.01}<7.31$, then $F>\lambda_{\alpha=0.01}$, namely, the linear regression is highly significant.

(3) Prediction interval According to mathematical statistical theory, the approximate prediction interval with the confidence coefficient $1-\alpha=0.95$ of the predicted value $\delta_{0}$ of $\delta$ at $T=T_{0}$ based on the regression Eq. 7 is $\left(\delta_{0}-2 \sigma\right.$, $\left.\delta_{0}+2 \sigma\right)$. The approximate prediction interval with the confidence coefficient $1-\alpha=0.99$ of the predicted value $\delta_{0}$ of $\delta$ at $T=T_{0}$ is $\left(\delta_{0}-3 \sigma, \delta_{0}+3 \sigma\right)$. Where, $\sigma$ is the variance of the predicted values.

$$
\sigma^{2}=\left[\sum_{i=1}^{n}\left(\delta_{i}-\bar{\delta}\right)^{2}-a \sum_{i=1}^{n}\left(\delta_{i}-\bar{\delta}\right)\left(T_{i}-\bar{T}\right)\right] /(n-2)=0.59
$$

Namely, $\sigma=0.77$. In the above equation, $\bar{\delta}$ is the average value of the measured radon exhalation rates; $\delta_{i}$ is the $i^{\text {th }}$ value of the measured radon exhalation rate; $\bar{T}$ is the average value of the measured temperature; $T i$ is the $i^{\text {th }}$ value of the measured temperature; $a$ is the monomial coefficient of the regression equation, 0.3657 ; $n$ is the sample size, 56 .

Whereby, the approximate prediction interval with the confidence coefficient $1-\alpha=0.95$ of the predicted value $\delta_{0}$ is $\left(\delta_{0}-1.54, \delta_{0}+1.54\right)$, while the approximate prediction interval with the confidence coefficient $1-\alpha=0.99$ of the predicted value $\delta_{0}$ is $\left(\delta_{0}-2.31, \delta_{0}+2.31\right)$.

\section{Impact of Mechanical Ventilation on Radon Exhalation Rate}

The measurement of radon exhalation rate of tunnel in the mechanical ventilation with the dynamic method should meet the following conditions. (1) Radon exhalation in tunnel should be uniform and the radon concentrations on the same section are equal. (2) The mechanical ventilation quantity is constant. To meet the above conditions, a fixed fan is selected in the experiment for the mechanical ventilation in the tunnel, and the measurement of various data in the tunnel is implemented after the stable operation of ventilation system for 24 hours.

\subsection{Experimental methods and measures}

Select the studied tunnel of about $120 \mathrm{~m}$ long. The overall measurement layout is shown in Fig. 7. According to the measurement principle of dynamic method as well as the calculation formulas of radon exhalation rate (4), not only the surface area and length of the tunnel shall be measured, but it is necessary to measure the ventilation quantities and radon concentrations in the two sections. In order to ensure the reliability of measurement results, the following methods and measures are applied during measurement.

(1) Tunnel selection In order to reduce the effects of changes in outer weather conditions on the measured data in the tunnel, the experimental section is located $100 \mathrm{~m}$ away from the tunnel entry, with a total length of $100 \mathrm{~m}$, namely the shaded area in Fig. 7.

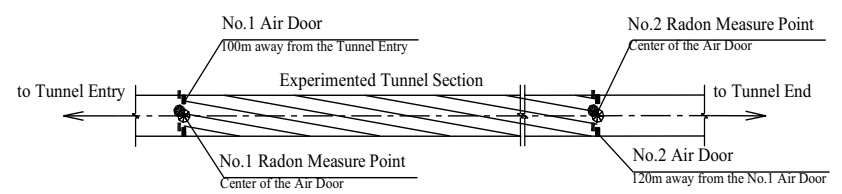

Fig. 7 Overall measurement layout of radon exhalation rate in mechanical ventilation case

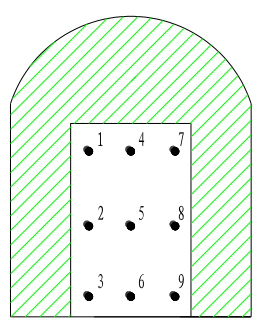

Fig. 8 Positions of wind speed measurement points in the mechanical ventilation section

(2) Ventilation system design and air distribution The ventilation system in tunnel is equipped with the single-wind-path rigid duct. On the duct, two fans of the same type are connected in parallel: one of which can achieve the blowing ventilation, namely the airflow direction in tunnel is outward from inward area when this fan operates; the other of which can achieve the exhaust ventilation, namely the airflow direction in tunnel is inward from outward area when this fan operates. Therefore, the research on the radon exhalation can be conducted separately in the blowing ventilation and exhaust ventilation.

The ventilation duct extends out of tunnel entry approximately $30 \mathrm{~m}$ and the duct mouth is in an open space. The impact of air backflow in the mechanical ventilation on the radon concentration in tunnel can be ignored. The duct in the tunnel is throughout the entire selected section, and its end is about $200 \mathrm{~m}$ away from the air door. The impact of duct mouth on the flow field in the tunnel can be ignored.

(3) Air volume measurement Considering that the natural ventilation quantity in the mechanical ventilation may result in the difference of total ventilation quantity in 
the sections of two ends of selected tunnel, it is necessary to separately measure the air volume in two sections. It is difficult to calculate the area of the cross section through all-section measurement due to the irregularity of the real tunnel section. In order to facilitate measurement and calculation, two air doors are separately set up at both ends of the selected tunnel (Fig. 7) as the measurement sections of wind speeds. A total of 9 measurement points are set up on the section (Fig. 8), and the average value of results form the 9 points is taken as the sectional wind speed. Finally, the total ventilation quantity shall be calculated according to the sectional wind speed in the section and the ventilation area.

(4) Measurement of radon concentration After mechanical ventilation, a large amount of radon flow out of tunnel due to the exhaust effect of ventilation and the distribution of radon concentration changes due to the dilution effect of ventilation. The experimental results show that, in the mechanical ventilation, the radon concentration in tunnel gradually increases along the flow direction and the maximum radon concentration will be at the end of airflow ${ }^{[7]}$. There are some similar conclusions in literature [8].

The air door changes the tunnel shape and affects the airflow in tunnel. A vortex will be formed when the air flows through the air door ${ }^{[9]}$, seriously affecting the uniformity of radon distribution in tunnel.

According to the above situation, in the mechanical ventilation, it is necessary to separately measure the radon concentrations at both ends of tunnel; the radon concentration in the center of air door can be considered to represent the concentration level of radon in the air flowing through the air door. The layout of radon detector in mechanical ventilation is shown in Fig. 7.

(5) Monitor of temperature outside tunnel

(6) Calculate the radon exhalation rate based on equation (4) according to the radon concentration and air volume at the air door.

\subsection{Data processing and analysis}

\subsubsection{Calculation of radon exhalation rate}

According to Eq.4, radon exhalation rate is calculated in accordance with the following equation:

$$
\delta=\frac{C\left(q x_{0}+Q\right)-C_{0} Q}{s x_{0}}
$$

Where, $C$ is the concentration of radon in the air flowing through the air door, $\mathrm{Bq} / \mathrm{m}^{3}$, where the external air door is referred to in the case of blowing ventilation and External air door in the case of exhaust ventilation; $C_{0}$ is the initial concentration of radon in the air flowing through the air door, $\mathrm{Bq} / \mathrm{m}^{3}$, where the External air door is referred to in the case of blowing ventilation and external air door in the case of exhaust ventilation; $Q$ is mechanical ventilation quantity through the air door, $\mathrm{m}^{3} / \mathrm{s}$, where the External air door is referred to in the case of blowing ventilation and external air door in the case of exhaust ventilation; $\left(q x_{0}+Q\right)$ is the total air volume through the air door, $\mathrm{m}^{3} / \mathrm{s}$, where the external air door is referred to in the case of blowing ventilation and External air door in the case of exhaust ventilation; $x_{0}$ is the length of the measured tunnel, taking $120 \mathrm{~m} ; s$ is the cross-section perimeter of tunnel, taking the average value of $12 \mathrm{~m}$.

\subsubsection{Data Analysis in blowing ventilation}

(1) Regression analysis Obtain 109 groups of effective data in blowing ventilation. The scatter diagram of temperature and radon exhalation rate is shown in Fig. 9, which indicates an obvious linear relationship. Therefore, the linear fitting is conducted.

After linear regression analysis, the linear regression equation of radon exhalation rate is

$$
\delta=1.7814 T-28.94
$$

Where, $\delta$ is the radon exhalation rate, $\mathrm{Bq} /\left(\mathrm{m}^{2} . \mathrm{s}\right) ; T$ is the temperate outside tunnel, $15^{\circ} \mathrm{C} \leq T \leq 34^{\circ} \mathrm{C}$

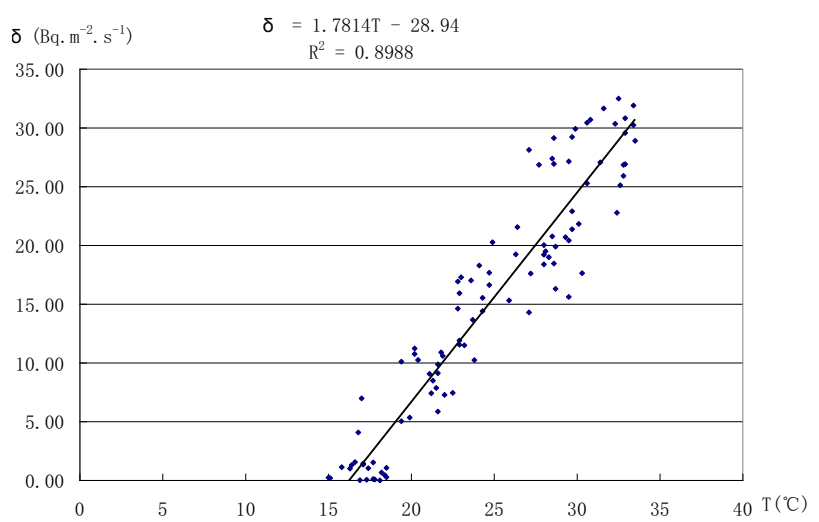

Fig. 9 Radon exhalation rate VS the temperature outside tunnel in blowing ventilation case

(2) Significance test Test the significance of the linear regression with the $F$ testing method. The test result shows that the linear regression is highly significant.

(3) Prediction interval The approximate prediction interval with the confidence coefficient $1-\alpha=0.95$ of the predicted value $\delta_{0}$ is $\left(\delta_{0}-6.74, \delta_{0}+6.74\right)$, while the approximate prediction interval with the confidence coefficient $1-\alpha=0.99$ of the predicted value $\delta_{0}$ is $\left(\delta_{0}-10.11\right.$, $\left.\delta_{0}+10.11\right)$.

\subsubsection{Data analysis in exhaust ventilation}

(1) Regression Analysis Obtain 89 groups of effective measurement data in exhaust ventilation. The scatter diagram of temperature and radon exhalation rate is shown in Fig. 10, which indicates an obvious linear relationship. Therefore, the linear fitting is conducted. After linear regression analysis, the linear regression equation of radon exhalation rate is

$$
\delta=2.8979 T-45.168
$$

Where, $\delta$ is the radon exhalation rate, $\mathrm{Bq} /\left(\mathrm{m}^{2} . \mathrm{s}\right) ; T$ is the temperate outside tunnel, $15^{\circ} \mathrm{C} \leq T \leq 25^{\circ} \mathrm{C}$. 


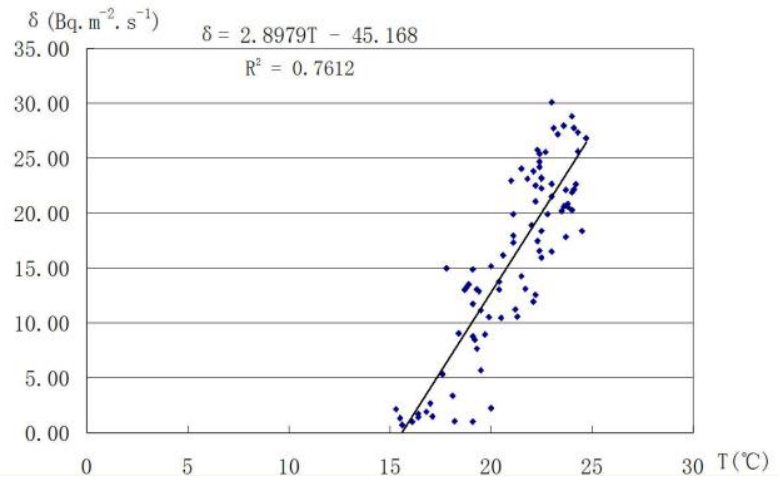

Fig. 10 Radon exhalation rate VS the temperature outside tunnel in exhaust ventilation case

(2) Test of linear regression significance After test with the $F$ testing method, the linear regression is proved to be highly significant.

(3) Prediction interval The approximate prediction interval with the confidence coefficient of 0.95 of the predicted value $\delta_{0}$ is $\left(\delta_{0}-8.22, \delta_{0}+8.22\right)$, while the approximate prediction interval with the confidence coefficient of 0.99 of the predicted value $\delta_{0}$ is $\left(\delta_{0}-12.33\right.$, $\left.\delta_{0}+12.33\right)$.

\subsubsection{Analysis of effects of two ventilation styles on radon exhalation rate}

It can be seen from the data analysis that, with the same ambient temperature, the radon exhalation rate in exhaust ventilation is higher than that in blowing ventilation. According to the above regression equation, if $k_{1}$ and $k_{2}$ are respectively assumed as the slopes of Eq.9 and Eq. 10 , then $\left(k_{2}-k_{1}\right) / k_{1} \approx 0.63$, meaning that the radon exhalation rate in exhaust ventilation is $63 \%$ higher than that in blowing ventilation. This result is slightly higher than that of research on some tunnel in literature [10]: the radon exhalation space density in exhaust ventilation is $40 \%-60 \%$ higher than that in blowing ventilation. The reason for this difference may be the higher permeability of over-broken rock in the experimented tunnel.

\section{Conclusions and Recommendations}

Based on the on-site experiments, this paper separately studies the regularity of radon exhalation in summer natural ventilation and two mechanical ventilations and achieves the following conclusions:

(1) In natural ventilation, the correlation between the radon exhalation rate in tunnel and the natural ventilation quantity as well as the external ambient temperature is linear. In the summer natural state, the radon exhalation rate is positively correlated with the ambient temperature, namely, the radon exhalation rate increases with the ambient temperature. The higher ambient temperature will cause the higher radon concentration in tunnel.

(2) In mechanical ventilation, the correlation between the radon exhalation rate in tunnel and the ambient temperature outside tunnel is linear. At the same ambient temperature, the radon exhalation rate in exhaust ventilation is $63 \%$ higher than that in blowing ventilation. The results show that under the same conditions, the exhaust ventilation can better accelerate the radon exhalation in rock than the blowing ventilation. Therefore, the blowing ventilation in tunnel is more conducive to radon control.

Based on the above conclusions, it is recommended to avoid working in over-broken granite tunnel and other underground constrictions when there is radon pollution during high temperature period in summer. If available, it is suggested to operate in early spring, late autumn or winter. When the mechanical ventilation is needed in the underground work space, it is necessary to select the blowing ventilation as the main ventilation style.

\section{References}

1. Z.Si-ming. Uranium and Radon Ventilation. Uranium Mining and Metallurgy, 4 (1992)

2. Frédeéric Perrier, Patrick Richon, Umesh Gautam, et al. Seasonal variations of natural ventilation and radon-222exhalation in a slightly rising dead-end tunnel. Journal of Environmental Radioactivity, 97 (2007)

3. LI Ren-jie. Determination of radon reduction rate and exploration on its influence factors. Uranium Mining and Metallurgy, 19 (2000)

4. Wu Hui-shan. Radon Measurement and Practical Data. Atomic Energy Press, (2001)

5. Zhao Yi-hui. Mine ventilation and air conditioning.: China University of Mining and Technology press, (1990)

6. Du Wei-xin. The Mine Ventilation Technology. China University of Mining and Technology press, (2009)

7. YE Yong-jun, DING De-xin, ZHOU Xing-huo, et al. Study on the maximal driving length of dummy drift in uranium mines. Chinese Journal of Nuclear Science and Engineering, 29 (2009)

8. André Unger, Stefan Finsterle, Gudmundur S. Bodvarsson. Estimating large-scale fractured rock properties from radon data collected in a ventilated tunnel. PROCEEDINGS TOUGH Symposium. Lawrence Berkeley Lab, Berkeley, Calif. .(2003)

9. Cai Zeng-ji, Long Tian-yu. Fluid Mechanical Pump and Fan (The Fifth Edition).China Architecture \& Building Press. (2009)

10. Zhu Si-ming, Calculation Method of Foreign Uranium Tunnel Air. China Nuclear Society Symposium on Radiation Environment of Mine, 1 (1994)

11. LIU Yong, ZHANG Xin-hua. Permeation and control of radon and its radiation products in underground mining of uranium deposits. China Mining Magazine, 13.(2004)

12. LI Ming, ZHANG Xin-hua, LIU Yong. Research on the regularity of emission and dispersion of radon in tunnel construction. Modern Tunneling Technology, 42 (2005) 